\title{
Fog and Atmospheric Turbulence in FSO: Models to mitigate
}

\author{
Ritu Gupta*, Ankita Aggarwal \\ Chandigarh Engineering College, Landran, Mohali \\ * ritu.4366@cgc.edu.in
}

\begin{abstract}
Free space optics (FSO) is an attractive technique and provides reliability in comparison to radio frequency (RF). The FSO provides high data rate capability and has high bandwidth efficiency. The FSO provides various advantages to radio frequency and it is operable at rain also. But as other techniques FSO also has some issues like absorption, scintillation and scattering, which degrades the quality of the signal. There are various models which estimates the losses due to fog and absorption. In this paper we have analysed the kim, kruse and gamma gamma - gamma turbulence modelin terms of atmospheric attenuation or visibility
\end{abstract}

Keywords: FSO, kim, kruse, fog,attenuation.

\section{INTRODUCTION}

Optical FSO is wireless optical communication in which the light is travelled through air. The light is generated through a source which can be LASER or a LED. The light is then amplified and transmitted through air. At the receiver side, the signal is received using a photo detector. However, while in signal transmission due to the air interface many interferences are added but they can be removed. In spite of it the most important reason for signal degradation in FSO is fog. Due to the fog the signal is absorbed. This is the most important reason of signal loss. FSO is based on line of sight propagation due to which any obstruction between transmitter and receiver in the form of fog or storm can become a major setback to the system[1-2]. Both RF and FSO technologies possess different characteristics and having their own compensation and disadvantage. RF communication be unsuccessful during heavy rainfall. Whereas, FSO communication unsuccessful during fog.

The complementary nature of these technologies used together in hybrid FSO/RF communication system to provide good signal quality to the user [3]. Various studies have been done for evaluate the performance of FSO communication system. It has been found that the quality factor (QF) of FSO-WDM system decreases with the increase in distance [4]. Section 2 focuses on the atmospheric losses due to attenuation. In section 3, different attenuation models have been compared. Finally, the paper is over and done with in section 4 .

\section{ATMOSPHERIC LOSSES}

Different atmospheric conditions such as haze, fog, rain etc. effect the performance of the signal as compared in clear weather conditions [5]. The losses in FSO are due to scintillation, scattering of light or absorption due to different atmospheric conditions. The photons in optical light, when travelling through atmosphere interacts with the atmospheric photons due to which the photons may be either absorbed or scattered. Moreover, in dense fog the photons while interacting with the dense water molecules gets extinguished. This also causes power losses due to which the strength of the signal decreases [6-7]. The signal attenuation is the major drawback of the FSO system as shown in equation 1 .

$$
\Gamma(\lambda)=\alpha 1(\lambda) \alpha 2(\lambda) \beta 1(\lambda) \beta 2(\lambda)
$$

where $\alpha 1$ and $\alpha 2$ are the absorption coefficient, $\beta 1$ and $\beta 2$ are the scattering coefficient.

2.1Absorption: Absorption is caused when the molecule in the atmosphere is interacted with the photons molecules which are transmitted through the air. When a photon is absorbed, an amount of heat is released. This depends upon the gas concentration in the atmosphere.

2.2Scattering: The photon gets scatter in the atmosphere due to fog which causes the distribution of the optical field of the photon again and again [8-10]

\section{VARIOUS MODELS IN FOG}

Practically, due to fog visibility reduces. The fog causes bad effects on the optical signal. However, the effect which the fog causes depends upon the density of the fog. In other words, it can be stated that the dense fog and the moderate fog causes different effects on the optical signal and uniquely they differently attenuate the optical signal. This effect can be well understood by taking different models in consideration [10-13]. Attenuation depends upon the visibility and is given by equation 2.

$$
\sigma=(3.91 / \mathrm{V}) *(\lambda / 550)^{-\mathrm{q}}
$$

where, $\lambda$ is wavelength and $\mathrm{q}$ depends uponthe visibility for different models.

3.1Kim model:The Kim model is used to find attenuation of the signal due to the fog[14].The Kim model has a fixed value of $\mathrm{q}$, which shows that as the $\lambda$ raise, the attenuation lessen. Value of $\mathrm{q}$ as in equation 1 is as per table 1 shown below:

Table 1:Value of q according to kim model [7]

\begin{tabular}{|l|l|}
\hline $\begin{array}{l}\text { Visibility range in } \\
(\mathrm{km})\end{array}$ & Value of $\mathrm{q}$ \\
\hline$>50$ & 1.6 \\
\hline $6<\mathrm{v}<50$ & 1.3 \\
\hline $1<\mathrm{v}<6$ & $.016 \mathrm{v}+0.34$ \\
\hline $0.5<\mathrm{v}<1$ & $\mathrm{v}-0.5$ \\
\hline$<0.5$ & 0 \\
\hline
\end{tabular}

The attenuation coefficient has been checked as per visibility coefficient at different wavelengths i.e. $850 \mathrm{~nm}, 950 \mathrm{~nm}$ and $1550 \mathrm{~nm}$. It can be observed from the figure 1 that the $1550 \mathrm{~nm}$ wavelength gives minimum attenuation. 


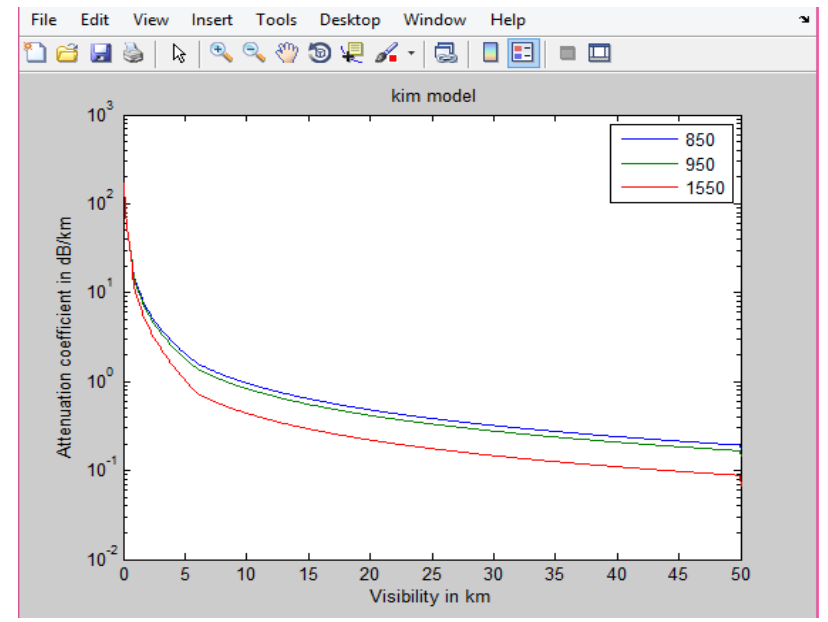

Fig1: Attenuation coefficient vs visibility curve of kim model

3.2Kruse model: The Kruse model is similar to Kim model [1516]. However, originally the Kruse model was intended for dense or thick fog. Because of which it is not suitable for visibility $\leq 1 \mathrm{KM}$. According to Kruse model value of $\mathrm{q}$ as in equation 1 is as per table 2 shown below:

Table 2: Value of $q$ according to Kruse model [7]

\begin{tabular}{|l|l|}
\hline $\begin{array}{l}\text { Visibility range in } \\
\mathrm{KM}\end{array}$ & Value of $\mathrm{q}$ \\
\hline$>50$ & 1.6 \\
\hline $6<\mathrm{v}<50$ & 1.3 \\
\hline $0<\mathrm{v}<6$ & $0.585 \mathrm{v}^{\wedge} 1 / 3$ \\
\hline
\end{tabular}

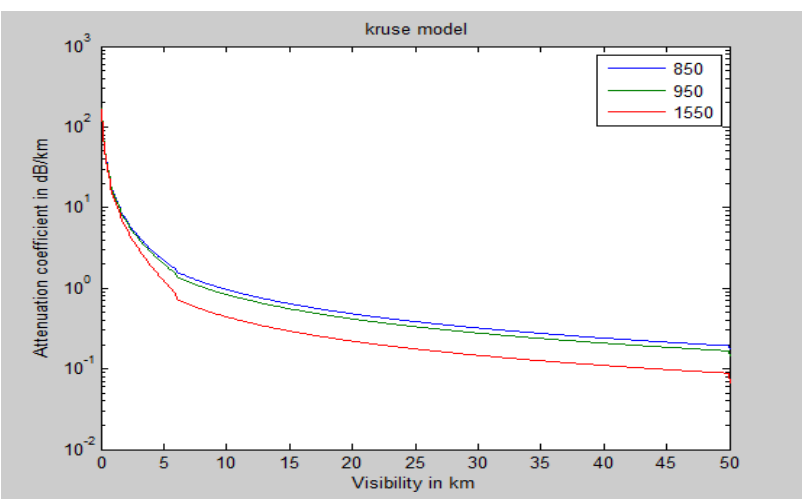

Fig2: Attenuation coefficient versus visibility of Kruse model

Again it can be observed figure 2that like in Kim model, the attenuation at $1550 \mathrm{~nm}$ is minimum as compared to $850 \mathrm{~nm}$ and $950 \mathrm{~nm}$.

3.3Gamma gamma turbulence model: Turbulences are caused due to different temperature of the earth surface and surrounding which cause turbulences in the air. The turbulences cause eddies in the atmosphere which changes the amplitude of the signal and causes power losses, the gamma model determines such turbulences effect on the propagating optical signal by taking in to consideration weak strong and moderate turbulences[17]. The unconditional gamma-gamma distribution function (GDF) is obtained as in equation 3 :

$\mathrm{GDF}=2(\alpha \beta)^{((\alpha+\beta) / 2)} / \Gamma(\alpha) \Gamma(\beta) \mathrm{I}^{((\alpha+\beta) / 2)-1} \mathrm{~K}_{\alpha-\beta} 2(\alpha \beta \mathrm{I})^{1 / 2}, \mathrm{I}>0$

where, $\alpha$ and $\beta$ represents the effective number of large and small scale eddies and are related to the index of refraction. $\Gamma($.) represents the gamma function. I denotes the irradiance of eddies due to large scale turbulence. Values of $\alpha$ and $\beta$ for different turbulence strength is

$$
\begin{array}{cc}
\text { Weak } & \alpha=11.6, \beta=10.1 \\
\text { Moderate } & \alpha=4, \beta=1.9 \\
\text { Strong } & \alpha=4.2, \beta=3.5
\end{array}
$$

For different strengths of turbulence such as week, moderate and strong given below the results in figure 3 , figure 4 and figure 5 respectively. The ensuing irradiance pdf takes the form of a general $\mathbf{K}$ distribution which is termed as gamma-gamma distribution from equation 3. The gamma-gamma pdf are determined for different turbulence conditions and it has been observed from the results that during strong turbulence the irradiance is very less.

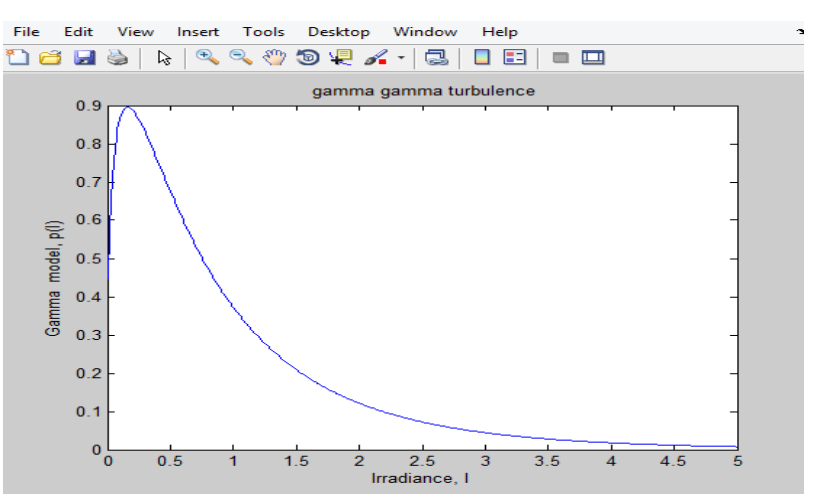

Fig 3: Gamma gamma turbulence model for strong turbulence

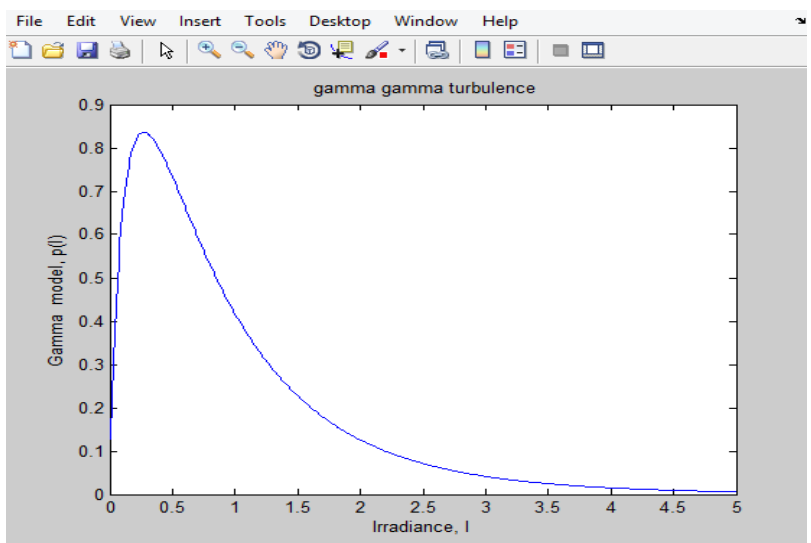

Fig 4: Gamma gamma turbulence model for moderate turbulence

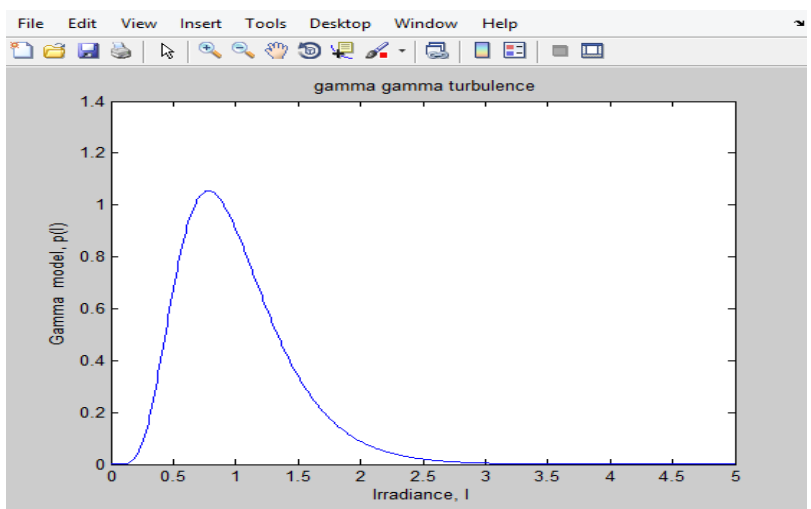

Fig 5: Gamma gamma turbulence model for strong turbulence 


\section{CONCLUSION}

In this paper, it has been shown that during fog, $1550 \mathrm{~nm}$ wavelength range is suitable for FSO communication as a result of less BER. The other pros and cons of FSO has also been discussed. It has been defined with taking in to consideration atmospheric losses and the weather condition especially the fog which severely effect the performance of FSO system. The performance of Kim and Kruse model for fog has been analyzed and it is concluded that at $1550 \mathrm{~nm}$ the attenuation-visibility curve gives best performance. Also, the effects of turbulence in the atmosphere have been analyzed using gamma-gamma turbulence model. It has been practical from the study that FSO has a brilliant future. A variety of advancements can be made in order to increase its bandwidth and efficiency. Alternate option to improve visibility is to make the light brighter. But this is not promising because the lights then become hazardous to the eyes. As long as laser or FSO communication is used within its capabilities, it will turn out to be the preferred high-bandwidth wireless technology for telecommunication. It is the way out to the issue of last mile bandwidth bottleneck.

\section{REFERENCES:}

[1]. Hennes Henniger, Otakar Wilfert' An Introduction to Free-space Optical Communications" Radio Engineering, Vol. 19, No. 2, June 2010.

[2]. Willebrand, H., Ghuman, B. Fiber optics without fiber. IEEE Spectrum, Vol. 38, No. 8, pp. 40 - 45, 2001

[3]. Ritu Gupta, Preeti Singh, "Hybrid FSO - RF System: A Solution to Atmospheric Turbulences in Long Haul Communication" International Journal of Scientific \& Engineering Research, Vol. 5, No. 11, pp 602-605, Nov. 2014.

[4]. Dheeraj duvey, Ritugupta, "Analysis of Fog Attenuation Models for Multi-Transceiver FSO System for Different Frequencies", Vol. 3, No. 6, pp. 216-220, June 2014.

[5]. Dheeraj duvey, Ritugupta, "Review Paper on Performance Analysis of A Free Space Optical System" International Journal of Application or Innovation in Engineering \& Management Vol. 3, No. 6, pp 135-139, June 2014.

[6]. K. W. Fischer, M. R. Witiw, and E. Eisenberg, "Optical attenuation in fog at a wavelength of 1.55 micrometers," Atmospheric Research, vol. 87, pp. 252-258, 2008.

[7]. I. I. Kim, B. McArthur, and E. Korevaar, "Comparison of laser beam propagation at $785 \mathrm{~nm}$ and $1550 \mathrm{~nm}$ in fog and haze for optical wireless communications," in Proc. SPIE 4214, Boston, MA, USA, 2001.

[8]. Z.Ghaseemlooy, W. Poopola, and S. Rajbhandri, "Optical wireless communications, system and channel modelling with matlab," CRC Press, London, UK, 2012.

[9]. Manor, H., Arnon, Sh.,"Performance of an optical wireless communication system as function of wavelength," Applied Optics, Vol. 42 No. 21, July 2003.

[10]. M.Grabner and V. Kvicera, "The wavelength dependent modelof extinction in fog and haze for free space opticalcommunication," J. of Optics Express, Vol. 19, pp. 33793386,2012

[11]. F. Nadeem, T. Javornik, E. Leitgeb, V. Kvicera, and G.Kandus, "Continental fog attenuation empirical relationshipfrom measured visibility data," of Radio Engineering, Vol. 19, No. 4, 2010.

[12]. Mazin Ali A. Ali "Analysis of Data Rate for Free Space Optical Communications System" IJECT, Vol. 5, Issue Spl-1, Jan March 2014.

[13]. R. M. Pierce, J. Ramapras, and E. C. Eisenberg, "Optical attenuation in fog and clouds"," Proc. SPIE 4530, Vol. 58, 2001.

[14]. Keiser, G., optical fiber communications, McGraw-Hill Company, 2000.

[15]. Bloom, S., Korevaar, E., Shuster, J., and Willebrand, H., understanding the performance of free-space optics, journal of networking, Vol. 2, pp. 178-200, 2003.

[16]. T. S. Rappaport. Wireless Communications - Principles and Practice. Prentice Hall, New Jersy, 1999.

[17]. W.Gappmair and M. Flohberger, "Error performance of coded FSO links in turbulent atmosphere modeled by gamma-gamma distributions," IEEE Transactions on Wireless Communications, Vol. 8, pp. 2209-2213, 2009 